\section{Hodgkin's Disease-Related Central Nervous System Angiopathy Presenting as Reversible Posterior Leukoencephalopathy}

Key words: Hodgkin's disease, central nervous system angiitis, reversible posterior leukoencephalopathy syndrome (RPLS)

Reversible posterior leukoencephalopathy syndrome (RPLS) is a clinical syndrome characterized by headache, conscious disturbance, seizure, and cortical visual loss with neuroimaging finding of edema in the posterior regions of the brain, with a reversible course (1). The precise pathomechanism of RPLS is not understood. However, association with uncontrolled hypertension, renal failure, eclampsia, or immunosuppressive agents has been implicated (1). We describe herein a case of Hodgkin's disease (HD)related central nervous system (CNS) angiitis with neuroimaging finding suggestive of RPLS. The pathophysiology of RPLS in cases with CNS angiitis is discussed.

For editorial comment, see $\mathrm{p} 900$.

A 20-year-old previously healthy woman developed diffuse headache with fever and visited a local hospital. The provisional diagnosis was infectious meningoencephalitis and she was accordingly treated with intravenous acyclovir and cephoperazone sodium. However, her state of consciousness deteriorated in the next few weeks, and she was transferred to our hospital 4 weeks after admission to the local hospital.

On admission, her body temperature was $37.5^{\circ} \mathrm{C}$, pulse rate $100 / \mathrm{min}$, and blood pressure $162 / 100 \mathrm{mmHg}$. The patient was in a semicomatose state with mild meningeal signs. Optic fundi were normal. Pupils were normal in size and equal, and reacted to light promptly. The patient retracted her extremities to noxious stimuli, but less movements were noted on the right side. Deep tendon reflexes were generally hyperactive with bilateral Babinski's sign. Routine hematology and biochemistry tests were normal. Autoantibodies and serological testing for organisms including herpes simplex virus, human immunodeficiency virus, and varicella-zoster virus were negative. Brain magnetic resonance imaging (MRI) showed extensive cerebral swelling and scattered T2 hyperintensities, with bilateral diffuse edema of the occipital subcortical white matter suggesting RPLS (Fig. 1A). No contrast enhancement was noted. Lumbar puncture revealed an opening pressure of $560 \mathrm{mmH}_{2} \mathrm{O}$; the cerebrospinal fluid was clear and colorless and contained 24 lymphocytes/ $\mu 1,1$ neutrophil/ $\mu \mathrm{l}$, protein $20 \mathrm{mg} / \mathrm{dl}$, and glucose $67 \mathrm{mg} / \mathrm{dl}$; herpes simplex virus DNA was negative; culture yielded no organism; and the cytological examination was negative. At that time, cerebral venous sinus thrombosis was suspected based on the elevated cerebrospinal fluid pressure, and cerebral angiography was performed. However, the venous system was normal. Instead, it showed segmental narrowing in the right internal carotid artery and in the branches of bilateral anterior and middle cerebral arteries (Fig. 1B). Posterior cerebral arteries were normal.

CNS angiitis was suspected based on the angiographic findings, and high dose steroid therapy was started. Intracranial pressure (ICP), which was monitored with a sensor inserted into the right frontal lobe, ranged between 200 to $250 \mathrm{mmH}_{2} \mathrm{O}$. However, in the first few days, it intermittently rose to as high as $500 \mathrm{mmH}_{2} \mathrm{O}$ for a few minutes. This rise of ICP occurred concomitant with abrupt elevation of arterial blood pressure, which was triggered by intratracheal suctioning or a passive change in body position. On the 10th hospital day, the state of consciousness began to improve, accompanied by reduction of the cerebral swelling and scattered T2 hyperintensities, and disappearance of the occipital edema on MRI.

Further investigation of the primary cause of CNS angiitis was conducted. Computed tomography scan of the body revealed enlarged lymph nodes in the upper mediastinum and bilateral supraclavicular fossa, and histopathological examination of a lymph node from the right supraclavicular fossa confirmed HD, nodular sclerosis. The patient received three courses of chemotherapy (doxorubicin hydrochloride $30 \mathrm{mg}$, bleomycin hydrochloride $13 \mathrm{mg}$, vinblastine sulfate $8 \mathrm{mg}$, and dacarbazine $500 \mathrm{mg}$ for each course) and 36 Gray of radiation therapy toward the cervical and mediastinum lymph nodes. The neurological signs continued to improve with clinical remission of $\mathrm{HD}$, and the patient achieved almost full recovery with only mild residual memory disturbance and generalized hyperreflexia.

In the present case, we considered that the encephalopathy was causally related to $\mathrm{HD}$, as our extensive investigation showed that HD was the only underlying illness and the therapies against HD induced further neurological improvements. Further, we presumed that vascular wall inflammation was the pathological correlate of the angiographic finding, although we could not histopathologically confirm such correlation. Similarly, 14 cases of HD-related CNS angiitis have previously been reported and a causative association has been considered $(2,3)$.

The interesting finding in our case was the bilateral occipital edema seen on the MRI suggesting RPLS. RPLS associated with systemic angiitis or isolated CNS angiitis have been reported $(1,4,5)$. Most of these cases were associated with marked hypertension, and the blood pressure elevation 

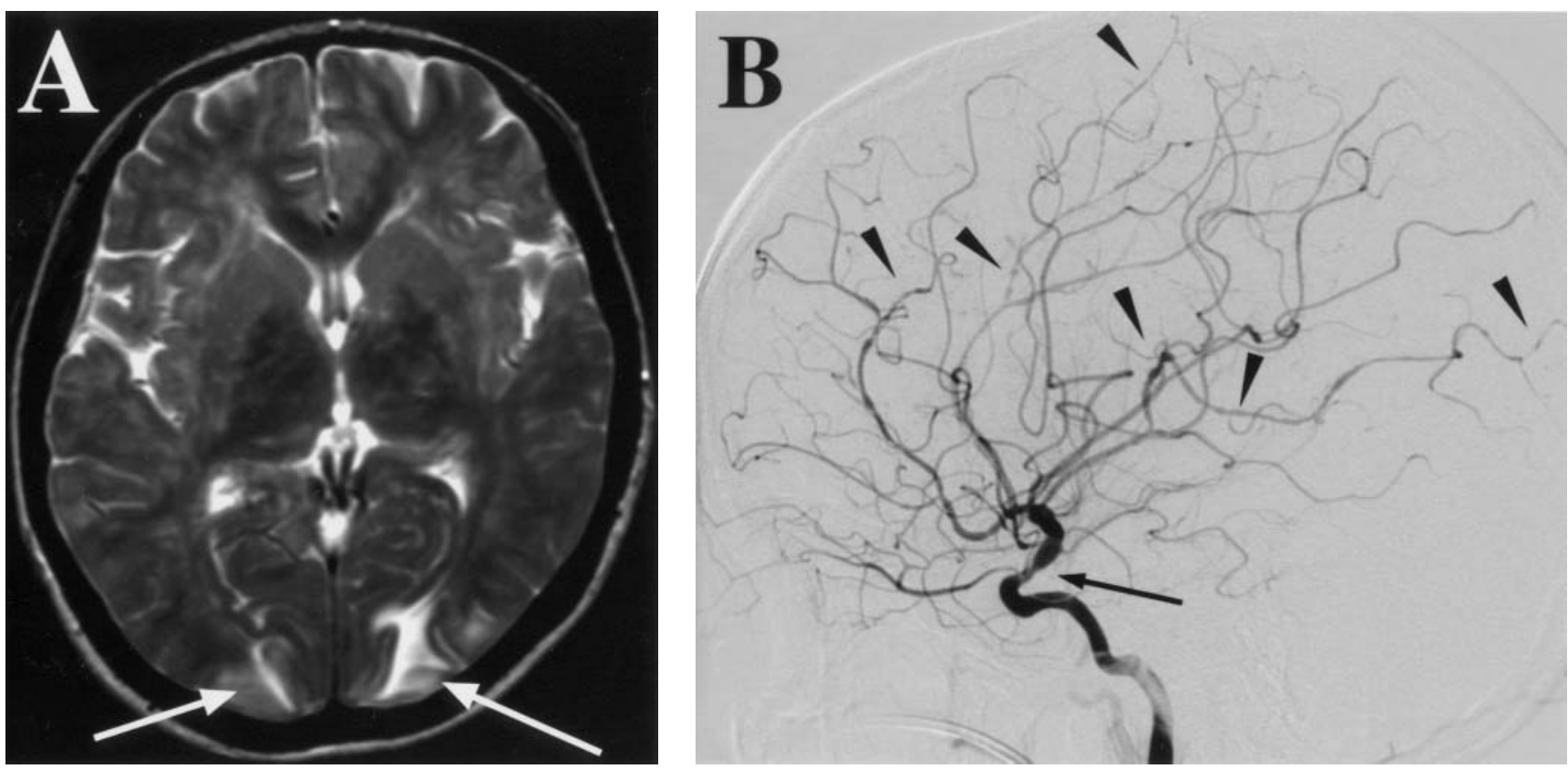

Figure 1. (A) T2-weighted MRI of the brain (TR 3,500 ms, TE $90 \mathrm{~ms}$ ) shows generalized cerebral swelling with slit-like ventricles, and scattered hyperintensities in the white matter, with diffuse edema of both occipital lobes suggesting reversible posterior leukoencephalopathy (white arrows). (B) Lateral view of the right internal carotid arteriography showing a focal narrowing in the supraclinoid segment (arrow) and segmental narrowing and beading in the branches of anterior and middle cerebral arteries (arrowheads).

beyond the autoregulatory capability of brain vasculature may be responsible as suggested for RPLS associated with hypertensive encephalopathy (1). Furthermore, vascular endothelial injuries due to inflammation were also considered to be associated with the fluid leakage resulting in RPSL (4). In addition, systemic fluid retention and hypertension induced by corticosteroids were considered relevant in some cases, even though corticosteroids have been reported to be effective for RPLS (4). In our case, the systemic hypertension alone was not sufficient to cause RPLS, and the vascular endothelial injury may have been responsible, although other unknown effects of HD could not be ruled out.

On the other hand, unusual rise in ICP was observed in the present case. Although cerebral swelling was seen in the MRI, it is inconceivable that the increase in intracranial tissue volume alone caused ICP elevation, since it lasted only a few minutes. Rather, as the ICP elevation was seen concomitant with abrupt elevation of systemic blood pressure, it is reasonable to speculate that systemic blood pressure elevation secondarily caused ICP elevation, which was accelerated by a disturbance of cerebral vessel autoregulation due to inflammation of the vascular wall. Since cerebral vessel dysautoregulation is considered one of the important factors in the pathogenesis of RPLS (4), we considered that this cerebral vessel dysautoregulation was also causally related to the MRI finding suggesting RPLS.

The underlying pathophysiology of RPLS is considered multifactorial. However, our case suggests that, in addition to hypertension, both increase in vascular permeability and disturbance in cerebral vessel autoregulation due to the vascular wall inflammation may be responsible for RPSL in cases with CNS angiitis.

Yusei MiYAZAKI, Yasutaka TAJIMA, Kazumasa Sudo, Akihisa Matsumoto, Jun TASHIRO*, Seiji KIKUCHI*, Hidenao SASAKI* and Kunio TASHIRO**

The Department of Neurology, Sapporo City General Hospital, Sapporo, *the Department of Neurology, Hokkaido University Graduate School of Medicine, Sapporo and **the Health Sciences University of Hokkaido School of Psychological Science, Sapporo

Received for publication January 13, 2004; Accepted for publication June 24, 2004

Reprint requests should be addressed to Dr. Yusei Miyazaki, the Department of Neurology, Hokkaido University Graduate School of Medicine, Kita-15 Nishi-7, Kita-ku, Sapporo 060-8638

\section{References}

1) Hinchey J, Chaves C, Appignani B, et al. A reversible posterior leukoencephalopathy syndrome. N Engl J Med 334: 494-500, 1996.

2) Rosen CL, DePalma L, Morita A. Primary angiitis of the central nervous system as a first presentation in Hodgkin's disease: A case report and review of the literature. Neurosurgery 46: 1504-1508; discussion 1508-1510, 2000.

3) Sheehy N, Sheehan K, Brett F, Kay E, Grogan L, Delanty N. Hodgkins disease presenting with granulomatous angiitis of the central nervous 
system. J Neurol 250: 112-113, 2003.

4) Kawano H, Kimura E, Ishizaki M, et al. Reversible posterior leukoencephalopathy syndrome in two patients with microscopic polyarteritis nodosa. Rinsho Shinkeigaku (Clin Neurol) 42: 949-953,
2002 (in Japanese, Abstract in English).

5) Wijdicks EFM, Manno EM, Fulgham JR, Giannini C. Cerebral angiitis mimicking posterior leukoencephalopathy. J Neurol 250: 444-448, 2003. 